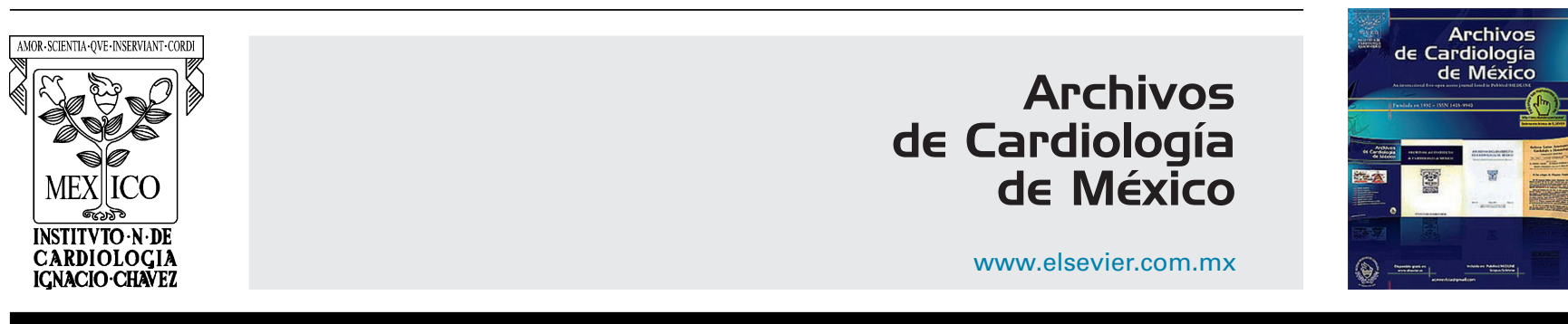

INVESTIGACIÓN CLÍNICA

\title{
Análisis de mortalidad y estancia hospitalaria en cirugía cardiaca en México 2015: datos del Instituto Nacional de Cardiología
}

\author{
Alejandra Rodríguez-Hernández ${ }^{\mathrm{a}, *}$, Martha García-Torres ${ }^{\mathrm{b}}$, Eduardo Bucio Reta ${ }^{\mathrm{a}}$ \\ y Francisco Martín Baranda-Tovar ${ }^{a}$
}

a Terapia Intensiva Cardiovascular, Instituto Nacional de Cardiología Ignacio Chávez, Ciudad de México, México

b Servicio Social en Investigación, Instituto Nacional de Cardiología Ignacio Chávez, Ciudad de México, México

Recibido el 22 de marzo de 2017; aceptado el 15 de noviembre de 2017

\author{
PALABRAS CLAVE \\ Mortalidad; \\ Cirugía cardiaca; \\ Cirugía valvular; \\ Estancia hospitalaria; \\ Choque cardiogénico; \\ México
}

\begin{abstract}
Resumen
Objetivo: Analizar las causas de mortalidad hospitalaria de pacientes operados de cirugía cardiaca en México en el año 2015. Identificar los factores de riesgo de mortalidad y realizar una correlación con el tiempo de estancia hospitalaria en terapia intensiva cardiovascular.

Método: Estudio de casos y controles. Se estudió la base de datos de la Terapia Intensiva Cardiovascular del Instituto Nacional de Cardiología. Se incluyeron todos los pacientes adultos operados de cirugía cardiaca en el año 2015.

Resultados: Se operaron 571 pacientes. La indicación quirúrgica predominante fue la cirugía de cambio valvular único o múltiple, seguida de la cirugía de revascularización coronaria y corrección de cardiopatías congénitas del adulto. La mortalidad global fue de un $9.2 \%$ y el $8 \%$ falleció en terapia intensiva. Los principales factores de riesgo de muerte fueron la presencia de falla orgánica o hipertensión pulmonar prequirúrgica, y el tiempo prolongado de circulación extracorpórea. La principal causa de muerte fue el choque cardiogénico. La mortalidad hospitalaria observada en esta población fue mayor para los operados de tromboendarterectomía pulmonar, cirugía de enfermedad aórtica compleja y cirugía valvular.

Conclusiones: La mortalidad de los pacientes operados de cirugía cardiaca en México difiere levemente de la reportada en la literatura mundial porque se trata mayormente de cirugía multivalvular y de procedimientos quirúrgicos mixtos complejos.

(c) 2017 Instituto Nacional de Cardiología Ignacio Chávez. Publicado por Masson Doyma México S.A. Este es un artículo Open Access bajo la licencia CC BY-NC-ND (http://creativecommons. org/licenses/by-nc-nd/4.0/).
\end{abstract}

\footnotetext{
* Autor para correspondencia. Juan Badiano 1, Col. Belisario Domínguez, Sección XVI, Delegación Tlalpan, 14080, Ciudad de México. México. Teléfono: 55-73-29-11. Ext. 1504.

Correo electrónico: alejandrarh1985@gmail.com (A. Rodríguez-Hernández).
} 


\section{KEYWORDS}

Mortality;

Cardiac surgery;

Valve surgery;

Hospital stay;

Cardiogenic shock;

Mexico
Analysis of mortality and hospital stay in cardiac surgery in Mexico 2015: Data from the National Cardiology Institute

\begin{abstract}
Objective: To analyse hospital mortality in patients subjected to cardiac surgery in Mexico during the year 2015, and identify the mortality risks factors, and its correlation with days of hospital stay in the cardiovascular intensive care unit.

Method: The database of Cardiovascular Intensive Care of the National Institute of Cardiology was examined for this cases and controls study that included only adult patients subjected to cardiac surgery during the year 2015.

Results: A total of 571 patients were subjected to a surgical procedure. The predominant indication was single or multiple valve replacement surgery, followed by coronary revascularisation surgery, and correction of adult congenital heart disease. Overall mortality was 9.2 , and $8 \%$ died in intensive care. The main risk factors for death were preoperative organ failure or pulmonary hypertension, and prolonged time with extracorporeal circulation. The primary cause of death was secondary to cardiogenic shock. The hospital mortality observed in this population was higher for patients undergoing pulmonary thromboendarterectomy, complex aortic disease surgery, and valvular surgery.

Conclusions: The mortality of patients undergoing cardiac surgery in Mexico differs slightly from that reported in the world literature, primarily because there were more multivalvular surgeries and mixed complex procedures performed.

(C) 2017 Instituto Nacional de Cardiología Ignacio Chávez. Published by Masson Doyma México S.A. This is an open access article under the CC BY-NC-ND license (http://creativecommons. org/licenses/by-nc-nd/4.0/).
\end{abstract}

\section{Introducción}

El reporte americano de la base de datos de la Society of Thoracic Surgeons, la Adult Cardiac Surgery Database, informó de la distribución de cirugía cardiaca en Estados Unidos de América y Canadá como sigue: cirugía de revascularización aislada, 69\%, cirugía de cambio valvular aórtico (CVAo), 13\%, cirugía de CVAo más revascularización coronaria $(\mathrm{RC}), 8 \%$, cirugía de cambio valvular mitral (CVM), $3 \%$, reparación de válvula mitral (RVM), $4 \%$, CVM y $\mathrm{RC}, 1 \%$, RVM y RC, $2 \%$. Aproximadamente un $23 \%$ fueron procedimientos combinados que incluyeron cirugía de aorta, CVM y CVAo, cambio o reparación valvular tricuspídea, cirugía para corrección de arritmias, implante de asistencia ventricular y reparación de defecto septal cardiaco. La mortalidad operatoria encontrada fue de un $9.8 \%$ para pacientes con CVM y RC, de un $2.2 \%$ para la cirugía de RC aislada, de un 2.1\% para el CVAo aislado, de un 3.9\% para el CVAo y RC, de un $4.7 \%$ para el CVM, de un $1.1 \%$ para la RVM y de un 4.1\% para la cirugía de RVM y RC. Los pacientes con mayor mortalidad operados de CVM y RC presentaron una mayor estancia hospitalaria, ventilación prolongada, falla renal, fibrilación auricular, reoperación, mediastinitis y evento vascular cerebral ${ }^{1}$.

Los pacientes operados de cirugía cardiaca representan una población única entre aquellos críticamente enfermos debido a que habitualmente tienen una estancia corta en la Unidad de Cuidados Intensivos (UCl). Sin embargo, algunos pacientes experimentan complicaciones postoperatorias significativas y pasan un período de tiempo prolongado en la $\mathrm{UCl}$, lo cual incrementa la mortalidad de forma directamente proporcional en la mayoría de los estudios clínicos.
Los criterios de estancia prolongada en UCl después de cirugía cardiaca no están bien definidos y varían según la literatura desde 2 días hasta 2 semanas en el postoperatorio. Dependiendo de la definición utilizada, la mortalidad hospitalaria en estancia prolongada en UCI varía entre el $15-40 \%^{2}$.

Bashour et al. encontraron una mortalidad del 33\% después de cirugía cardiaca en pacientes que tuvieron una estancia en la UCI mayor de 10 días consecutivos. En el estudio de Elfstrom et al., la sobrevida a un año cayó hasta el $68 \%$ en aquellos pacientes que tuvieron una estancia mayor de 14 días en la $\mathrm{UCl}^{3,4}$.

\section{Métodos}

Se realizó un análisis de la base de datos de Terapia Intensiva Cardiovascular del Instituto Nacional de Cardiología Ignacio Chávez para identificar aquellos pacientes, hombres y mujeres mayores de 18 años de edad, ingresados en el postoperatorio inmediato de cirugía cardiaca, independientemente del tipo de cirugía realizada: cirugía de RC, cirugía de cambio valvular (incluyó cambio valvular único, doble y triple), cirugía de enfermedad aórtica compleja, cirugía de cardiopatías congénitas del adulto, tromboendarterectomía pulmonar y cirugía mixta en el año 2015 (enero de 2015-diciembre de 2015). Se realizó un análisis de mortalidad, comorbilidad preoperatoria y tiempos quirúrgicos para determinar los factores de riesgo de mortalidad en cirugía cardiaca. El diseño realizado fue un estudio retrospectivo observacional de casos y controles. 


\section{Análisis estadístico}

La fase descriptiva permitió conocer las características sobresalientes en este estudio en cuanto a la distribución de cada una de las variables, dependientes e independientes. Las variables de distribución normal se informan con media y porcentaje. En las variables sin distribución gaussiana se utiliza la prueba de Wilcoxon después de aplicar estadísticos comparativos convencionales, siempre que lo permitiera la relevancia práctica de los grupos creados. Se utilizó el software Stata12.1 para Mac.

\section{Resultados}

En 2015 se operaron 571 pacientes de cirugía cardiaca. La indicación quirúrgica predominante fue la cirugía valvular única o múltiple, seguida de la cirugía de RC y la cirugía de corrección de cardiopatías congénitas del adulto, con unos porcentajes de $48.5,18$ y $8 \%$, respectivamente (tabla 1 ).

Las indicaciones de cirugía se subdividieron de acuerdo con el tipo de internamiento para la misma, como: cirugía programada, cirugía de urgencia o cirugía de vía rápida. Esta última es un tipo de cirugía programada realizada con un internamiento denominado «vía rápida» para pacientes de bajo y moderado riesgo quirúrgico tomando como base criterios institucionales propios y criterios internacionalmente aceptados, implementada en el Instituto Nacional de Cardiología desde el año 1999. La tromboendarterectomía pulmonar y la cirugía de cardiopatías congénitas fueron las cirugías más frecuentemente realizadas con internamiento programado; esta última también fue la cirugía realizada más frecuentemente con internamiento por vía rápida. Las cirugías realizadas de forma urgente mayoritariamente fueron la cirugía de pericardio, la resección de tumores cardiacos y la cirugía de RC (tabla 2).

La mortalidad global encontrada fue de 53 pacientes (9.2\%); $46(8 \%)$ de ellos fallecieron en la UCI. La principal causa de muerte fue choque cardiogénico en el $42.8 \%$, choque séptico en el $21.43 \%$ ( $50 \%$ por neumonía nosocomial, $25 \%$ por mediastinitis y $25 \%$ por otras fuentes de infección); un $16.6 \%$ fallecieron por choque hipovolémico, $7 \%$ por choque mixto y $12.3 \%$ por otras causas, las cuales incluyen trombosis y necrosis mesentérica y coagulación intravascular diseminada (fig. 1).

En la descripción de las características de los pacientes fallecidos (tabla 3), claramente se puede observar que los que fallecieron en la UCI tenían diagnóstico de neumopatía prequirúrgica documentada como EPOC o diferente a EPOC y además nefropatía, tuvieron mayor hipertensión pulmonar y tiempos quirúrgicos prolongados, con un promedio de 235 min de circulación extracorpórea y 138 min de pinzamiento aórtico. El tiempo máximo de circulación extracorpórea en los pacientes finados fue de $588 \mathrm{~min}$, con un tiempo mínimo de 0 min para aquellas cirugías cardiovasculares que no utilizaron bomba de circulación extracorpórea.

En la tabla 4 se puede observar la mortalidad hospitalaria de acuerdo con el tipo de cirugía. La cirugía reportada con mayor mortalidad por el número de casos analizados fue la tromboendarterectomía pulmonar (fallecimiento de un paciente de un total de 5 operados), seguida de la cirugía de enfermedad aórtica compleja y la cirugía valvular. De los pacientes valvulares operados finados, a un $40.6 \%$ se les había realizado cirugía de doble cambio valvular, a un $12.5 \%$, cirugía de cambio valvular único y a un $9.3 \%$, cirugía de triple cambio valvular, y un $37.5 \%$ fue operado de cirugía de cambio valvular único o múltiple más otro procedimiento quirúrgico que incluyó cirugía de enfermedad aórtica compleja, RC y cirugía de pericardio.

El porcentaje de mortalidad significativamente alta en cirugía de enfermedad aórtica compleja corresponde a las cirugías realizadas de forma urgente (58\%).

En el análisis del tiempo de estancia hospitalaria, los pacientes finados en la $\mathrm{UCl}$ tuvieron una mayor estancia hospitalaria (4.5 días) en comparación con los que sobrevivieron (1.8 días) y esto mostró diferencias estadísticamente significativas $(p=0.01)$.

\section{Discusión}

En la UCl del Instituto Nacional de Cardiología se encontró un porcentaje de mortalidad hospitalaria similar al reportado en la literatura médica internacional para todos los tipos de cirugía, sin embargo, con un incremento ligeramente mayor para los pacientes operados de cirugía valvular y cirugía de RC, los cuales se reportaron en el año $2015: 7.9$ y $4.9 \%$ vs.

Tabla 1 Indicaciones de cirugía cardiaca en el año 2015 en el Instituto Nacional de Cardiología

\begin{tabular}{lc}
\hline Tipo de cirugía & Total (porcentaje) \\
\hline Valvular & $277(48.5)$ \\
Revascularización coronaria & $102(18)$ \\
Trasplante cardiaco & $1(0.17)$ \\
Cirugía de pericardio & $17(3)$ \\
Cirugía de enfermedad aórtica compleja o cirugía de aorta abdominal & $29(5)$ \\
Cardiopatías congénitas del adulto & $46(8)$ \\
Resección de tumores & $9(1.5)$ \\
Tromboendarterectomía pulmonar & $5(1)$ \\
Cirugía cardiaca mixta & $44(7.7)$ \\
Otros procedimientos cardiovasculares & $8(1.4)$ \\
Cirugía no cardiovascular & $33(5.7)$ \\
Total de cirugías & $571(100)$ \\
\hline
\end{tabular}


Tabla 2 Tipo de indicación quirúrgica, catalogada como electiva, urgente o vía rápida

\begin{tabular}{|c|c|c|c|c|}
\hline \multirow[t]{2}{*}{ Tipo de cirugía } & \multicolumn{3}{|c|}{ Motivo de cirugía } & \multirow[t]{2}{*}{ Total } \\
\hline & Electiva & Urgencia & Vía rápida & \\
\hline Valvular & $147(53)$ & $110(39.7)$ & $20(7.2)$ & $277(100)$ \\
\hline Revascularización coronaria & $36(35.2)$ & $66(64.7)$ & $0(0)$ & $102(100)$ \\
\hline Trasplante cardiaco & $0(0)$ & $1(100)$ & $0(0)$ & $1(100)$ \\
\hline Cirugía de pericardio & $1(6)$ & $16(94)$ & $0(0)$ & $17(100)$ \\
\hline Enfermedad aórtica compleja o cirugía de aorta abdominal & $11(38)$ & $17(58.6)$ & $1(3.4)$ & $29(100)$ \\
\hline Cardiopatías congénitas del adulto & $26(56.5)$ & $4(8.6)$ & $16(35)$ & $46(100)$ \\
\hline Resección de tumores & $2(22.2)$ & $7(77.7)$ & 0 & $9(100)$ \\
\hline Tromboendarterectomía pulmonar & $4(80)$ & $1(20)$ & 0 & $5(100)$ \\
\hline Cirugía cardiaca mixta & $16(36.3)$ & $26(59)$ & $2(4.5)$ & $44(100)$ \\
\hline Otros procedimientos cardiovasculares ${ }^{a}$ & $1(12.5)$ & $7(87.5)$ & $0(0)$ & $8(100)$ \\
\hline Cirugía no cardiovascular ${ }^{\mathrm{b}}$ & $15(45.4)$ & $18(54.5)$ & $0(0)$ & $33(100)$ \\
\hline Total de cirugías & $259(45.3)$ & $273(47.8)$ & $39(6.8)$ & $571(100)$ \\
\hline
\end{tabular}

a Otros procedimientos cardiovasculares incluyeron la retirada de cables de marcapasos y DAl, y la colocación de marcapasos.

b Las cirugías no cardiovasculares incluyen colocación de catéter de diálisis, cesárea, exploración mediastinal, nefrectomía y trasplante renal.

\section{CAUSAS DE MORTALIDAD \%}

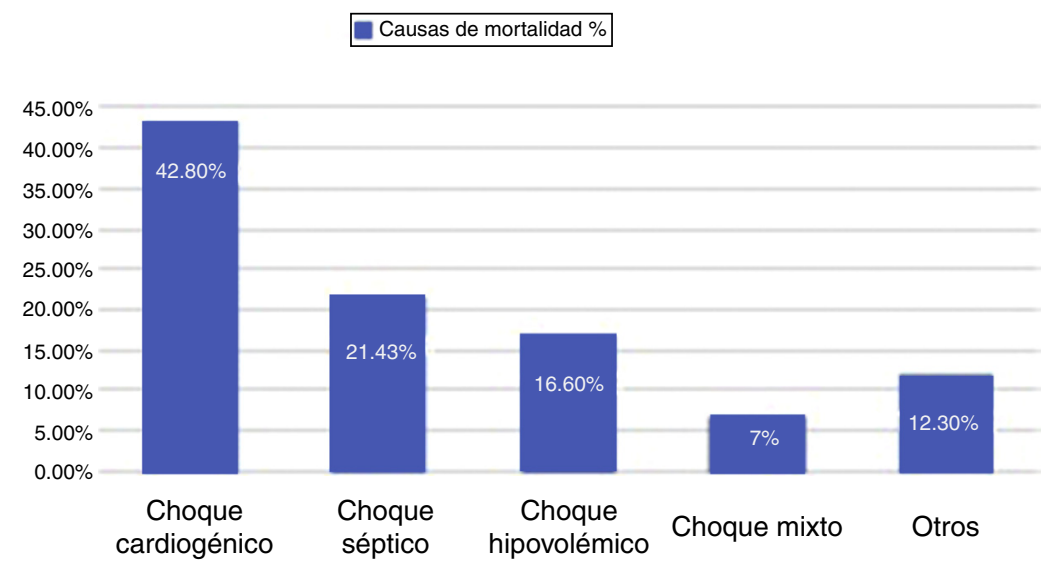

Figura 1 Causas de muerte de los pacientes operados de cirugía cardiaca en 2015.

Fuente: Base de datos del Instituto Nacional de Cardiología.

3.4 y $2.3 \%$ en el mundo, haciendo énfasis en que este porcentaje de mortalidad en lo que respecta a cirugía valvular corresponde a la cirugía de una sola válvula y el porcentaje de mortalidad mundial en RC implica cirugía únicamente dirigida a la revascularización.

La mortalidad reportada en América del Norte es de aproximadamente un 4-10\% para los procedimientos mixtos que implican CVAo, además de RC y CVM, además de RC. En nuestra serie la mortalidad en cirugía valvular fue de un $7.9 \%$, sin embargo, prácticamente el $50 \%$ eran procedimientos mixtos, incluyendo doble y triple cambio valvular.

El porcentaje de mortalidad en nuestra población se espera diferente, dado que somos un centro de referencia en México y América Latina para pacientes con diagnóstico de cardiopatía reumática inactiva. Aunque la fiebre reumática aguda ha declinado en Europa y Estados Unidos, la enfermedad persiste como la principal causa de morbimortalidad cardiovascular en las poblaciones de países en vías de desarrollo. Alrededor de un $25 \%$ de los pacientes tienen estenosis mitral aislada y en torno a un $40 \%$ doble lesión mitral, seguida de enfermedad multivalvular ${ }^{5,6}$.

En 1994, se estimaba que 12 millones de individuos sufrían cardiopatía reumática en el mundo. Una gran proporción de ellos requerían cirugía valvular en los siguientes 5-10 años. La mortalidad variaba de $0.5 / 100,000$ habitantes en Dinamarca a 8.2/100,000 habitantes en China o 1.8/100,000 habitantes en América Latina, con un estimado de 332,000 muertes en el mundo para el año $2000^{5}$.

Consideramos que el mayor porcentaje de mortalidad en cirugía valvular corresponde a la cirugía valvular múltiple, que implica un mayor tiempo de circulación extracorpórea y una mayor complejidad de la técnica quirúrgica.

El porcentaje reportado duplicado prácticamente para la cirugía de RC corresponde al porcentaje de mortalidad total, que incluye los procedimientos quirúrgicos mixtos complejos, en su mayoría cirugía de cambio valvular 
Tabla 3 Características demográficas de la población de estudio

\begin{tabular}{|c|c|c|c|}
\hline Variable & $\begin{array}{l}\text { Finados en } \mathrm{UCl} \\
\mathrm{n}=46\end{array}$ & $\begin{array}{l}\text { Pacientes vivos } \\
\mathrm{n}=525\end{array}$ & $\mathrm{p}$ \\
\hline Edad (años), media & 55.6 & 52 & NS \\
\hline \multirow{2}{*}{ Género, \% } & M 9.7 & M 90.3 & NS \\
\hline & H 6.7 & H 93.3 & \\
\hline \multicolumn{4}{|l|}{ Comorbilidad, \% } \\
\hline Diabetes & 19.5 & 26.8 & NS \\
\hline Hipertensión & 34.7 & 44.3 & NS \\
\hline Dislipidemia & 10.8 & 21.7 & NS \\
\hline Neumopatía o EPOC & 10.8 & 3.8 & 0.02 \\
\hline Hepatopatía & 0 & 0.5 & NS \\
\hline Nefropatía & 26 & 8 & 0.001 \\
\hline Infarto agudo del miocardio previo & 8.6 & 12.5 & NS \\
\hline FEVI menor de $40 \%$ prequirúrgica & 7.3 & 6.6 & NS \\
\hline \multicolumn{4}{|l|}{ Tiempo quirúrgico } \\
\hline PSAP $\geq 35 \mathrm{mmHg}, \%$ & 24 & 19.8 & 0.05 \\
\hline CEC $(\min )$, media & 235 & 119 & $<0.001$ \\
\hline PAo (min), media & 138 & 95 & $<0.001$ \\
\hline
\end{tabular}

Tabla 4 Tipo de cirugía y mortalidad en la Unidad de Cuidados Intensivos

\begin{tabular}{lc}
\hline Tipo de cirugía cardiaca & Mortalidad en UCI, \% \\
\hline Valvular & 7.9 \\
Revascularización coronaria & 4.9 \\
Cardiopatías congénitas del adulto & 4.3 \\
Cirugía de enfermedad aórtica compleja & 17 \\
Resección de tumores cardiacos & 0 \\
Cirugía de pericardio & 5.8 \\
Tromboendarterectomía & 20 \\
\hline
\end{tabular}

UCI: Unidad de Cuidados Intensivos.

único o múltiple, además de cirugía de pericardio, aorta, tumores o cardiopatías congénitas en el mismo tiempo quirúrgico.

En relación con los principales factores de riesgo para mortalidad hospitalaria, encontramos que la neumopatía EPOC o diferente a EPOC documentada prequirúrgica, la nefropatía documentada prequirúrgica, la hipertensión pulmonar, los tiempos quirúrgicos de circulación extracorpórea, el pinzamiento aórtico y la estancia hospitalaria en $\mathrm{UCl}$ son aquellos que tuvieron significación estadística.

Es esperado que la disfunción orgánica prequirúrgica signifique un mayor riesgo de mortalidad, pues tales datos se han demostrado ya en los grandes estudios que son sustento científico de las escalas utilizadas para valorar el riesgo de mortalidad en cirugía cardiaca, por ejemplo, el aclaramiento de creatinina y la necesidad de diálisis, o el diagnóstico prequirúrgico de EPOC en la escala EuroSCORE $1 \mathrm{I}^{7,8}$.

En cuanto a los días de estancia hospitalaria, comprobamos que los pacientes finados en la $\mathrm{UCl}$ tienen un mayor tiempo de internamiento -de 4.5 días- frente a los los pacientes que sobreviven y son egresados a piso -1.8 días-.

La mortalidad más alta encontrada según el tipo de cirugía fue para la tromboendarterectomía pulmonar, la cirugía de enfermedad aórtica compleja y la cirugía valvular, todas estas con indicación quirúrgica urgente.

\section{Conclusiones}

La mortalidad en México de los pacientes operados de cirugía cardiaca es similar a la reportada en el mundo. Difiere en la cirugía valvular y la RC discretamente porque en este artículo se reporta la mortalidad global, la cual implica que son procedimientos mixtos con mayor complejidad técnica y mayor circulación extracorpórea.

La estancia hospitalaria prolongada en la UCI se relaciona estrechamente con la mortalidad en dicha unidad y la mortalidad hospitalaria en pacientes operados de cirugía cardiaca. Los factores de riesgo encontrados para mortalidad, además de los días de estancia en $\mathrm{UCI}$, son: la EPOC o la neumopatía previa o la nefropatía previa documentada con o sin diálisis, la hipertensión pulmonar y los tiempos prolongados de circulación extracorpórea y pinzamiento aórtico.

\section{Financiación}

No se recibió patrocinio de ningún tipo para llevar a cabo este artículo.

\section{Conflicto de intereses}

Los autores declaran no tener ningún conflicto de intereses. 


\section{Bibliografía}

1. D'Agostino R, Jacobs JP, Badhwar V, et al. The Society of Thoracic Surgeons Adult Cardiac Surgery database: 2017 update on outcomes and quality. Ann Thorac Surg. 2017;103:18-24.

2. Yu P, Cassiere H, Fishbein J, et al. Outcomes of patients with prolonged Intensive Care Unit length of stay after cardiac surgery. J Cardiothorac Vasc Anesth. 2016;30:1550-4.

3. Bashour CA, Yared JP, Ryan TA, et al. Long-term survival and functional capacity in cardiac surgery patients after prolonged intensive care. Crit Care Med. 2000;28:3847-53.

4. Elfstrom KM, Hatefi D, Kilgo PD, et al. What happens after discharge? An analysis of long-term survival in cardiac surgical patients requiring prolonged intensive care. J Card Surg. 2012;27:13-9.
5. World Health Organization. Rheumatic fever and rheumatic heart disease. Report of a WHO Expert Consultation Geneva, 29 October-1 November 2001. WHO Technical Reports Series; 923. Geneva: WHO; 2001. p. 1-120.

6. Gewitz M, Baltimore R, Tani L, et al. Revision of the Jones criteria for the diagnosis of acute rheumatic fever in the era of Doppler echocardiography. A scientific statement from the American Heart Association. Circulation. 2015;131:1806-18.

7. Molina M. Estratificación del riesgo en cirugía cardiaca. Arch Cardiol Mex. 2002;72:141-7.

8. Carreño M. Escalas de riesgo en cirugía cardiaca: ¿qué predicen, para quién y para qué? Rev Col Cardiol. 2013;20:170-1. 